\title{
A propósito de uma crise convulsiva inaugural: um relato de caso
}

Marisa Monteiro Gomes, ${ }^{1}$ Susana Patrícia Leal Rebelo

\section{RESUMO}

Introdução: A crise convulsiva inaugural é uma causa frequente de recurso ao serviço de urgência. Estima-se que destes casos apenas 40 a 50\% tenham episódios recorrentes. Assim, face a uma crise convulsiva inaugural é importante excluir algumas condições que possam mimetizar o surgimento de convulsões.

Descrição do caso: Mulher, 45 anos, casada, com diagnósticos de mioma uterino, doença fibrocística da mama, perturbação de ansiedade e abuso de medicação (alprazolam). Em setembro de 2016 recorreu ao serviço de urgência por crise convulsiva tónico-clónica inaugural, sem aparentes fatores desencadeantes. Após exclusão de causas infeciosas, vasculares ou lesões ocupantes de espaço do sistema nervoso central teve alta clínica com o diagnóstico de crise epilética inaugural, medicada com levetiracetam.

Em janeiro de 2017 recorreu ao médico de família para mostrar nota de alta de neurologia. Quando questionada refere suspensão abrupta da toma de alprazolam alguns dias antes da crise convulsiva, apesar das indicações dadas de redução gradual do fármaco. Em março teve novo internamento por ideias delirantes e discurso incoerente após gastroenterite aguda. Teve alta com diagnóstico de episódio confusional agudo de etiologia orgânica/iatrogénica. Após a alta recorre ao médico de família e admitiu manter o consumo abusivo de alprazolam, que suspendeu repentinamente aquando da gastroenterite.

A utente não reconhecia o uso abusivo do fármaco. A família, apesar de ter sentido inicialmente dificuldades em lidar com a situação, acabou por ser um importante aliado no tratamento desta utente.

Comentário: Este caso clínico ilustra várias situações complexas na prática clínica, nomeadamente a dificuldade em compreender o utente como um todo no contexto de urgência, a ausência de um sistema de comunicação eficaz entre os diversos níveis de cuidados, a omissão de informação por parte dos doentes e o uso abusivo de certos fármacos.

Destaca a importância do envolvimento da família no tratamento e acompanhamento de diferentes situações clinicas, assim como o papel do médico de família que através do acompanhamento longitudinal do doente e de uma relação médico-doente privilegiada melhor o conhece.

Palavras-chave: Benzodiazepinas; Síndroma de abstinência; Convulsão; Médico de família.

\section{INTRODUÇÃO}

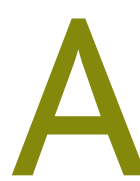
crise convulsiva inaugural é uma das causas de recurso ao serviço de urgência - cerca de $1 \%{ }^{1}$ Estima-se que destes casos apenas 40 a $50 \%$ tenham episódios recorrentes. A epilepsia é frequentemente diagnosticada após uma crise convulsiva inaugural, sendo uma das doenças neurológicas mais comuns. ${ }^{2}$ Contudo, erros no diagnóstico de epilepsia po-

1. Médica Interna de Medicina Geral e Familiar. USF Antonina, ACeS Ave Famalicão. 2. Médica Interna de Medicina Geral e Familiar. USF Nova Estação, ACeS Ave Famalicão. dem levar a decisões terapêuticas inapropriadas, com prescrição de anticonvulsivantes e custos desnecessários, que podem resultar em dano para o doente..$^{3-4}$

Assim, num doente com suspeita de uma crise convulsiva inaugural é importante excluir outras condições que possam mimetizar o surgimento de convulsões epiléticas. ${ }^{5-6}$ As crises convulsivas não epiléticas são frequentemente confundidas com epilepsia. Podem ser causadas por distúrbios fisiológicos ou psicológicos mas, ao contrário da verdadeira epilepsia, as convulsões não são consequência de descargas elétricas anormais no cérebro. ${ }^{7}$ Assim, é importante o diagnóstico diferencial 
com convulsões psicogénicas, alterações paroxísticas não epiléticas (como a síncope ou arritmias cardíacas) e convulsões provocadas, que são uma manifestação aguda a uma agressão cerebral e que geralmente não recorrem quando a causa subjacente é removida.

As benzodiazepinas (BZD) são psicofármacos com efeitos depressores ao nível do sistema nervoso central (SNC), utilizados para diversos fins terapêuticos, entre os quais a redução da ansiedade, tratamento da insónia, sedação, relaxamento muscular, indução de anestesia e abstinência alcoólica. ${ }^{8}$ As BZD pertencem ao grupo de fármacos mais consumidos em Portugal. ${ }^{9}$ Relativamente ao seu mecanismo de ação, facilitam a ação do ácido gama-aminobutírico (GABA), neurotransmissor inibidor do SNC, sobre os seus recetores. ${ }^{8}$

Todas têm o mesmo mecanismo de ação e efeitos adversos semelhantes. No entanto, diferem nas suas características farmacocinéticas, ou seja, início de ação e tempo de semivida. Sendo o alprazolam uma das BZD mais consumidas, tem um início de ação e uma semivida intermédias. ${ }^{8}$

O uso desta classe farmacológica condiciona uma vigilância apertada por parte do médico no sentido de evitar o abuso destas substâncias, bem como sintomas de ansiedade ou de abstinência que podem ocorrer com a interrupção abrupta da sua toma. A síndroma de abstinência refere-se ao conjunto de sintomas físicos e psicológicos presentes na descontinuação de uma substância. ${ }^{10}$ As suas manifestações clínicas aparecem mais ou menos rapidamente após a interrupção do fármaco. A cronologia das manifestações depende da cinética da molécula em questão: quanto menor o tempo de semivida mais intensas serão as manifestações da síndroma de abstinência. Os sintomas são bastante polimorfos, mas incluem ansiedade, irritabilidade, perturbações do sono, dores difusas, alterações sensoriais e do trato digestivo e hipotensão ortostática. ${ }^{8}$

Nas suas formas mais severas podem acompanhar-se de delírio psicótico, convulsões e alucinações (Quadro I). Vários estudos demonstraram que $20-100 \%$ dos doentes medicados com BZD por longos períodos de tempo sofrerão sintomas de síndroma de abstinência. ${ }^{11}$ Já outros estudos referenciam que os sintomas mais graves e as taxas de recaída são maiores em BZD de semivida curtas quando abruptamente retiradas, comparativamente às BZD de semivida longa. ${ }^{12}$

\begin{tabular}{|l|l|}
\hline $\begin{array}{l}\text { QUADRO I. Sintomas mais frequentes da síndroma de } \\
\text { abstinência das BZD (adaptação) }\end{array}$ \\
\hline \begin{tabular}{l} 
Sintomas ligeiros \\
\hline Físicos
\end{tabular} & Psiquiátricos \\
\hline Tremores & Irritabilidade \\
\hline Palpitações & Inquietação \\
\hline Sudorese & Agitação \\
\hline Letargia & Dificuldade de concentração \\
\hline Naúseas & Insónias \\
\hline Vómitos & Pesadelos \\
\hline Anorexia & Disforia \\
\hline Cefaleias & Falta de memória \\
\hline Sintomas gripais & Despersonalização \\
\hline Mialgias & \\
\hline
\end{tabular}

\section{DESCRIÇÃO DO CASO}

Isabel (nome fictício), 45 anos, residente em Famalicão, raça caucasiana, casada, $12^{\circ}$ ano de escolaridade, contabilista durante 22 anos, atualmente vendedora. Pertence a uma família nuclear na fase IV do ciclo de vida familiar de Duvall.

Apresenta, como antecedentes pessoais, mioma uterino, doença fibroquística da mama, perturbação de ansiedade e abuso de medicação (alprazolam).

Como antecedentes familiares destaca-se o pai com sequelas de acidente vascular cerebral e a mãe com diagnóstico recente de linfoma, sendo Isabel a cuidadora de ambos.

Encontra-se medicada habitualmente com alprazolam 1mg e desogestrel $75 \mu \mathrm{g}$.

Sem hábitos tabágicos ou alcoólicos.

A 14 de março de 2016 recorreu à sua médica de família (MF), em consulta programada, para mostrar ecografia endovaginal para vigilância de fibromioma uterino. Solicitou também receitas de alprazolam para si e para a sua mãe, dizendo que tinha vendas suspensas de três caixas na farmácia. Referia que o marido também tomava dos seus comprimidos por problemas relacionados com o trabalho. Posto isto, a MF questionou a doente acerca da posologia da medicação e do número de embalagens solicitadas nos últimos seis meses. A doente assumiu o abuso do alprazolam, referindo que 
tomava o fármaco quatro vezes por dia, porque "se tomar só um comprimido à noite ando muito nervosa, preciso de tomar mais quantidade para andar bem" e "esta situação da minha mãe e do meu pai estão a afetar-me muito", "sem esta medicação não consigo dormir". Negava sintomatologia depressiva, referia ansiedade e insónia intermédia. Foi aconselhada referenciação a consulta de psiquiatria e início de tratamento com antidepressivo, mas a utente recusou. Ao exame do estado mental apresentava-se ansiosa, com humor subdepressivo, sem ideação suicida e sem atividade heteróloga. Assim, a MF explicou-lhe os riscos do abuso da medicação, da necessidade de reduzir gradualmente a toma de alprazolam, tendo sido fornecido um esquema de redução ao longo de oito semanas. Optou-se por iniciar trazodona 150mg e referenciação a consulta de psicologia, tendo-se remarcado consulta para dentro de dois meses para reavaliar a situação.

A 23 de maio de 2016 a doente faltou à consulta marcada. Nessa semana, a mãe da doente, numa consulta de rotina com a MF, refere estar muito preocupada com a filha, dizendo que ela toma às escondidas o seu alprazolam. A MF explicou-lhe os riscos da toma abusiva de medicação e foi advertida para não dar mais da sua medicação à filha. A MF remarcou nova consulta para a Isabel, mas a utente faltou novamente. Faltou também à consulta de psicologia.

A 18 de setembro recorreu ao serviço de urgência (SU) do Centro Hospitalar de São João, após perda de consciência súbita, com os quatro membros em extensão e mordedura da língua, com a duração de cerca de três minutos e com amnésia para o sucedido. Negava alterações da fala, da força ou sensibilidade, vertigens, náuseas ou vómitos, cefaleias prévias ou posteriores ao episódio. Negava também sintomatologia cardíaca, pulmonar, febre ou sintomatologia compatível com infeção respiratória, urinária ou gastrointestinal prévia. Como medicação habitual referiu apenas toma de anticontracetivo oral.

Já no SU, às $23 \mathrm{~h}$, foi presenciada crise tónico-clónica generalizada, com confusão pós-ictal, que reverteu após medicação.

Realizou meios complementares de diagnóstico, entre os quais, estudo analítico sem alterações de relevo; eletrocardiograma com ritmo sinusal; radiografia torácica sem condensações ou infiltrados; punção lombar com pesquisa de ADN de herpes simplex 1 e 2 negativos e tomografia computorizada crânio-encefálica sem alterações de relevo. Dado este quadro foi internada para vigilância e investigação etiológica. Durante o internamento de três dias não teve recidiva das crises convulsivas. No prosseguimento da investigação etiológica realizou ainda eletroencefalograma e ressonância magnética cerebral que não mostraram alterações. Teve alta com diagnóstico de epilepsia e foi medicada com clobazan 10mg à noite e levetiracetam $500 \mathrm{mg}$ por dia. Foi orientada também para consulta de neurologia.

A 26 de janeiro de 2017 recorreu à MF, em consulta programada, para mostrar informação clínica do internamento no serviço de neurologia. Quando abordado o contexto da ocorrência das crises convulsivas, a doente refere que tinha suspendido abruptamente a toma de alprazolam alguns dias antes do sucedido, apesar das indicações de redução gradual feitas pela MF. Refere interrupção porque na farmácia não lhe cediam o alprazolam sem receita médica. Após a alta hospitalar reiniciou toma de alprazolam $1 \mathrm{mg}$ por dia e não iniciou o clobazan nem o levetiracetam. Discutiu-se a necessidade de encaminhamento para psiquiatria, mas a doente mostrou-se reticente e disse não precisar de ajuda.

Foi tentado contacto telefónico várias vezes com a colega de neurologia do Centro Hospitalar de São João para dar conhecimento do uso abusivo de BZD da doente, sem qualquer sucesso. Alternativamente optou-se por enviar email para o hospital ao cuidado da colega.

A 27 de fevereiro de 2017 recorreu ao SU do Centro Hospitalar Médio Ave (CHMA) por quadro clínico com dois dias de evolução de vómitos, diarreia e febre. Teve alta com o diagnóstico de gastroenterite aguda e cuidados sintomáticos.

No dia 3 de março de 2017 recorreu novamente ao SU do CHMA por quadro clínico de ideias delirantes e discurso incoerente, com início após a alta hospitalar a 28 de fevereiro. À admissão apresentava tremor irregular dos membros superiores e movimentos mioclónicos das mãos. Realizou estudo analítico, que revelou hipocaliemia $(2,9 \mathrm{mEq} / \mathrm{l})$, tomografia axial computorizada crânio-encefálica, sem alterações de relevo, e punção lombar que foi normal. Posteriormente foi transferida para o Hospital de Braga, tendo ficado internada para vigilância e continuação do estudo. Durante o internamento teve melhoria progressiva do quadro clíni- 
co, com desaparecimento das ideias delirantes e posteriormente das mioclonias e tremores das mãos. No prosseguimento do estudo realizou estudo analítico sem alterações, nova punção lombar normal, eletroencefalograma sem registo de atividade epileptiforme ou outras alterações e ressonância magnética cerebral sem alterações. Foi avaliada por psiquiatria, que considerou tratar-se de um episódio confusional agudo de etiologia orgânica/iatrogénica. Teve alta clínica medicada com valproato de sódio $500 \mathrm{mg}$, duas vezes por dia, e orientada para consulta externa de neurologia.

No dia 13 de março de 2017 foi a consulta à MF para mostrar nota de alta do serviço de neurologia do Hospital de Braga. Após ser questionada sobre o sucedido admite que estaria novamente a tomar abusivamente alprazolam e, no contexto do episódio de gastroenterite, terá suspendido o fármaco. O marido, que a acompanhava na consulta, refere que ela estaria a tomar o alprazolam dele até ao referido episódio. Referiu ainda que não conseguiu reduzir a toma de alprazolam nem iniciou trazodona, conforme prescrito. Após explicação da gravidade da toma da situação, que o marido desconhecia, e discussão com a doente e o marido acerca do uso abusivo deste fármaco, a utente acabou por concordar que precisa de ajuda para a resolução do seu problema e aceita encaminhamento para consulta de psiquiatria. Referia manter ansiedade marcada, insónia intermédia, humor deprimido e anedonia reativa aos problemas de saúde dos pais. Concordou iniciar tratamento com sertalina 50mg e trazodona $150 \mathrm{mg}$ e redução gradual da toma de alprazolam ao longo de oito semanas.

Foi dada informação clínica em carta para a doente levar aquando da consulta de neurologia no Hospital de Braga.

\section{COMENTÁRIO}

Este caso clínico ilustra várias situações complexas na prática clínica, nomeadamente a dificuldade em compreender o utente como um todo no contexto de urgência, frequentemente decorrente de constrangimentos informáticos, sendo por vezes difícil aceder aos cuidados do utente noutros níveis de saúde. Ilustra também a importância do MF como elemento detentor de informação clínica relevante acerca do paciente e as dificuldades de comunicação entre os cuidados de saúde primá- rios e secundários. Apesar de várias tentativas de comunicação pelo MF, a ausência de um sistema de comunicação eficaz entre os diversos níveis de cuidados pode condicionar, como no caso apresentado, um atraso no diagnóstico e consequências para a saúde do utente.

Destacam-se ainda as dificuldades que todos os médicos encontram na prática clínica diária, como a omissão de informação por parte dos doentes, as faltas às consultas, a não adesão à terapêutica e o uso abusivo de certos fármacos. O presente caso é uma fiel ilustração deste tipo de situações: a paciente não revelou ao neurologista nem o consumo abusivo de BZD nem a sua suspensão abrupta, que teria sido uma informação fundamental para o diagnóstico.

Destaca-se também a importância do envolvimento da família no tratamento e acompanhamento de diferentes situações clínicas. A família foi um elemento fundamental para a MF perceber melhor toda a situação. A mãe da utente revelou à MF que a filha tomava a sua medicação e referiu impotência em lidar com a situação. Adicionalmente, foi um importante aliado no processo de aceitação do problema por parte da doente e posterior plano de tratamento e acompanhamento.

Contudo, situações há que, devido ao sigilo profissional inerente, é impeditivo procurar ativamente o apoio da família.

Adicionalmente, alerta para a vigilância dos doentes sob BZD e para a importância da monitorização da duração do seu uso, tentando, sempre que possível, que o seu uso seja pelo menor período de tempo possível. O abuso de BZD é uma situação comum na prática clínica, que só é identificada se existir uma relação paciente-médico eficaz, em que haja confiança, abertura e sensibilidade para esta problemática. Por outro lado, a síndroma de abstinência de BZD num contexto de crises convulsivas é um diagnóstico etiológico a ponderar, depois de excluídas todas as causas mais frequentes de convulsões.

Assim, é fundamental que a sua suspensão seja realizada progressivamente ao longo de várias semanas. ${ }^{8}$

Evidencia ainda o papel do MF como o profissional que presta cuidados abrangentes e continuados aos indivíduos no contexto da sua família que, no caso apresentado, era fundamental no diagnóstico. O MFé aquele que, através deste acompanhamento longitudinal e da relação de confiança estabelecida com o paciente, melhor o conhece. 


\section{REFERÊNCIAS BIBLIOGRÁFICAS}

1. Martindale JL, Goldstein JN, Pallin DJ. Emergency department seizure epidemiology. Emerg Med Clin North Am. 2011;29(1):15-27.

2. World Health Organization. Epilepsy: fact sheet [homepage]. Geneva: WHO; 2019 Jun. Available from: https://www.who.int/en/news-room/ fact-sheets/detail/epilepsy

3. Begley CE, Famulari M, Annegers JF, Lairson DR, Reynolds TF, Coan S, et al. The cost of epilepsy in the United States: an estimate from population-based clinical and survey data. Epilepsia. 2000;41(3):342-51.

4. Krumholz A, Wiebe S, Gronseth G, Shinnar S, Levisohn P, Ting T, et al. Practice Parameter: evaluating an apparent unprovoked first seizure in adults (an evidence-based review): report of the Quality Standards Subcommittee of the American Academy of Neurology and the American Epilepsy Society. Neurology. 2007;69(21):1996-2007.

5. Hauser WA, Hesdorffer DC. Epilepsy: frequency, causes, and consequences. New York: Demos Publications; 1990. ISBN 9780939957323

6. Berg AT, Shinnar S. The risk of seizure recurrence following a first unprovoked seizure: a quantitative review. Neurology. 1991;41(7):965-72.

7. Krumholz A. Nonepileptic seizures: diagnosis and management. Neurology. 1999;53(5 Suppl 2):576-83.

8. Almeida M. Benzodiazepinas: da terapêutica ao abuso e dependência [dissertation]. Coimbra: Faculdade de Medicina da Universidade de
Coimbra; 2015.

9. Furtado C. Psicofármacos: evolução do consumo em Portugal Continental (2000-2012). Lisboa: INFARMED; 2013.

10. American Psychiatric Association. DSM-V: diagnostic and statistical manual of mental disorders. 5th ed. New York: APA; 2013. ISBN 9780890425558

11. Uzun S, Kozumplik O, Jakovljević M, Sedić B. Side effects of treatment with benzodiazepines. Psychiatr Danub. 2010;22(1):90-3.

12. Rickels K, Schweizer E, Case G, Greenblatt DJ. Long-term therapeutic use of benzodiazepines: I. Effects of abrupt discontinuation. Arch Gen Psychiatry. 1990;47(10):899-907.

\section{CONFLITO DE INTERESSES}

Os autores declaram não ter quaisquer conflitos de interesse.

\section{ENDEREÇO PARA CORRESPONDÊNCIA}

Marisa Monteiro Gomes

E-mail: marisamonteirogomes@gmail.com

http://orcid.org/0000-0002-0427-1778

Recebido em 18-03-2017

Aceite para publicação em 10-10-2018

\section{ABSTRACT}

\section{CONCERNING AN INAUGURAL CONVULSIVE CRISIS: A CASE REPORT}

Introduction: The inaugural convulsive crisis is a frequent cause of recurrence to the emergency department. It is estimated that only 40 to $50 \%$ of these cases have recurrent episodes. Thus, in the face of an inaugural convulsive crisis, it is important to exclude some conditions that may mimic the onset of convulsions.

Case description: Female, 45 years old, married, diagnosed with uterine myoma, fibrocystic breast disease, anxiety disorder and medication abuse (alprazolam). In September 2016, she went to the emergency department due to an inaugural tonic-clonic convulsive crisis, with no apparent triggering factors. After exclusion of infectious, vascular and space occupant lesions of the central nervous system, she was discharged with the diagnosis of an epileptic seizure with unknown etiology, medicated with levetiracetam.

In January 2017, she applied to the family physician to show the discharge note when questioned she admitted the abrupt withdrawal of alprazolam a few days before the seizure, despite indications of gradual reduction of the drug. In March she was hospitalized for delirious ideas and incoherent speech after acute gastroenteritis. She was discharged with the diagnosis of an acute confusional episode of organic/iatrogenic etiology. After the discharge, she went to the family physician and admitted to continuing abusing alprazolam, which she suddenly stopped when she had the gastroenteritis.

The patient did not recognize the abusive use of the drug. The family, although initially having difficulties in dealing with the situation, ended up being an important ally in the treatment of this patient.

Comment: This clinical case illustrates several complex situations in clinical practice, namely the difficulty in understanding the patient as a whole in the context of urgency, the absence of an effective communication system between the different levels of care, the omission of information by patients and the abuse of certain drugs.

It highlights the importance of the family involvement in the treatment and follow-up of different clinical situations, as well as the role of the family physician, who through the longitudinal follow-up of the patient and a better doctor-patient relationship better knows him.

Keywords: Benzodiazepines; Abstinence syndrome; Seizure; Family physician. 